\title{
Bu open Disabling chronic low back pain as an iatrogenic disorder: a qualitative study in Aboriginal Australians
}

\author{
Ivan B Lin, ${ }^{1,2}$ Peter B O'Sullivan, ${ }^{2}$ Juli A Coffin, ${ }^{3}$ Donna B Mak, ${ }^{4}$ Sandy Toussaint, ${ }^{5}$ \\ Leon M Straker
}

To cite: Lin IB, O'Sullivan PB, Coffin JA, et al. Disabling chronic low back pain as an iatrogenic disorder: a qualitative study in Aboriginal Australians. BMJ Open 2013;3:e002654.

doi:10.1136/bmjopen-2013002654

- Prepublication history for this paper are available online. To view these files please visit the journal online (http://dx.doi.org/10.1136/ bmjopen-2013-002654).

Received 30 January 2013 Revised 7 March 2013 Accepted 7 March 2013

This final article is available for use under the terms of the Creative Commons Attribution Non-Commercial 2.0 Licence; see http://bmjopen.bmj.com

For numbered affiliations see end of article.

\section{Correspondence to}

Ivan B Lin;

ivan.lin@cucrh.uwa.edu.au

\section{ABSTRACT}

Objectives: To determine the low back pain beliefs of Aboriginal Australians; a population previously identified as protected against the disabling effects of low back pain due to cultural beliefs.

Design: Qualitative study employing culturally appropriate methods within a clinical ethnographic framework.

Setting: One rural and two remote towns in Western Australia.

Participants: Thirty-two Aboriginal people with chronic low-back pain (CLBP; 21 men, 11 women). Participants included those who were highly, moderately and mildly disabled.

Results: Most participants held biomedical beliefs about the cause of CLBP, attributing pain to structural/ anatomical vulnerability of their spine. This belief was attributed to the advice from healthcare practitioners and the results of spinal radiological imaging. Negative causal beliefs and a pessimistic future outlook were more common among those who were more disabled. Conversely, those who were less disabled held more positive beliefs that did not originate from interactions with healthcare practitioners.

Conclusions: Findings are consistent with research in other populations and support that disabling CLBP may be at least partly iatrogenic. This raises concerns for all populations exposed to Western biomedical approaches to examination and management of low back pain. The challenge for healthcare practitioners dealing with people with low back pain from any culture is to communicate in a way that builds positive beliefs about low back pain and its future consequences, enhancing resilience to disability.

\section{INTRODUCTION}

Contemporary evidence for chronic low back pain (CLBP) recognises the biological, psychological and social influences on the natural history on the condition; the biopsychosocial model. ${ }^{1}{ }^{2}$ Within this framework the roles of CLBP beliefs and misperceptions have been gaining prominence as the

\section{ARTICLE SUMMARY}

Article focus

- The low back pain (LBP) beliefs of Aboriginal Australians with chronic LBP.

Key messages

- Contrary to previous research negative beliefs, including an anatomical/structural cause of pain and pessimistic future outlook, were common.

- Negative beliefs originated from interactions with healthcare practitioners suggesting disabling LBP may be partly iatrogenic.

- Biomedical-orientated management approaches to LBP are far reaching, highlighting the need for healthcare practitioners to positively influence beliefs as part of LBP care in all settings.

Strengths and limitations of this study

- The use of a qualitative and culturally secure research approach, and extended engagement with participants gave a unique insight into LBP beliefs among Aboriginal Australians and increased the rigour of the study.

- Findings were in line with research in other countries and cultural groups.

economic and social impacts of CLBP on Western $^{\mathrm{i}}$ societies have been increasing ${ }^{3}{ }^{4}$ and the failure of biomedical approaches to CLBP care is apparent. ${ }^{5}$ Negative beliefs about CLBP have been reported to predict CLBP disability, such as the perceptions of a biomedical cause of pain (eg, underlying structural/anatomical problem), that pain will be permanent or get worse in the future, and excessive fear of activity or movement out of concern of causing damage. ${ }^{2}{ }^{6-8}$ Misperceptions about CLBP such as these

'The authors recognise that use of the term 'Western' assumes one homogenous group regardless of region, sociality and cultural life. Here the term is used with caution to draw a distinction between Indigenous cultures and those arising primarily from non-Indigenous beliefs and practices, often designated 'Western'. 
are common in British, Norwegian and Canadian populations, ${ }^{9-11}$ as well as among healthcare practitioners such as medical practitioners, physiotherapists and chiropractors. ${ }^{12} 13$ By influencing or perpetuating negative beliefs, there is the potential for healthcare practitioners to have a negative impact on their patients disorder. Negative perceptions about CLBP reflect widespread sociocultural attitudes in Western societies and are hypothesised to underlie the 20th century 'epidemic' of CLBP disability. ${ }^{2}$

Despite suffering a tremendous burden of disease Aboriginal Australians have been uniquely identified as protected from the disabling effects of CLBP because of cultural beliefs. One study found that, despite a high prevalence of CLBP in one remote central Australian Aboriginal community, the impact of CLBP was small as few pain behaviours were observed and people did not seek healthcare. ${ }^{14}$ This reported limited impact of CLBP has been attributed to cultural beliefs where "[Aboriginal] people do not regard back pain as a health issue ${ }^{14}$; a conclusion that has been cited to illustrate the influence of culture on CLBP. ${ }^{2}$ It is likely that traditional Aboriginal cultural beliefs posit CLBP as of 'natural cause' (as opposed to environmental or supernatural) and are an accepted part of everyday life resulting in a temporary state of weakness, ${ }^{15}$ rather than a chronic illness which is disabling and unlikely to improve. However, this study was conducted in the early 1990's and the findings may not be valid for the contemporary situation.

If the conclusions of previous research are substantiated, valuable lessons for Western societies may be learned. However our recent research in regional and remote areas of Western Australia found that contrary to earlier findings, CLBP had substantial impacts on the lives of Aboriginal men and women, affecting emotional wellbeing, work, family and cultural participation. ${ }^{16}$ These impacts, when understood within the context of Aboriginal society, were profound for the Aboriginal men and women who were most disabled. ${ }^{16}$ Furthermore, although previous research has made certain claims about the CLBP beliefs of Aboriginal people ${ }^{14}$ these were not examined in detail. Therefore, the aim of the current study was to provide an in-depth exploration of back pain beliefs in Aboriginal people with CLBP and their relationship to disability.

\section{METHODS}

\section{Settings}

The research was undertaken in three towns in Western Australia; a regional town assigned the pseudonym of 'Regiontown'-population35 000, and two remote towns-'Goldstone' and 'Desertedge' with populations under 1000 and 500 people. Goldstone and Desertedge were classified as "very remote ${ }^{, 17}$ with limited accessibility to material goods and services. Medical emergencies would necessitate medical evacuation via the Royal Flying Doctor Service. In Regiontown, Aboriginal people were approximately $10 \%$ of all residents while the majority of residents in Goldstone and Desertedge were Aboriginal (Aboriginal and Torres Strait Islander people represent $2.5 \%$ of the Australian population and 3.8\% of the Western Australian population ${ }^{18}$ ). Each town includes culturally distinct Aboriginal language groups; however, there are extended sociocultural, economic and family networks across the region.

\section{Study approach, design and rationale}

This qualitative study was approached from an interpretive perspective, developing from the premise that the reality of CLBP is constructed by Aboriginal men and women who experience it, and their daily interactions in an immediate and wider social world. ${ }^{19} 20$ The framework of clinical ethnography ${ }^{21}$ was applied to 'understand the relationship between the lived experience of an illness (the bodily experience and know how) and the illness as domesticated and understood theoretically and 'scientifically' by medicine'. ${ }^{22}$ The position of the researchers was conceptualised as interpreting Aboriginal people's experiences within the context of contemporary understandings of CLBP, and the theoretical framework guiding the inquiry was a biopsychosocial model of CLBP. ${ }^{12}$

A second substantive methodological consideration was cultural security, which refers to processes that ensure that the research was conducted in a manner respectful of Aboriginal cultural values and beliefs. ${ }^{23} 24$ This consideration is essential for ethical purposes, ${ }^{25}$ improves data quality and ensures that the interpretation incorporates an Aboriginal cultural lens.

\section{Participants}

Eligible participants were Aboriginal adults with dominant pain in the low back region (T12 to gluteal fold) that had occurred episodically within a 6 -month period or lasted for more than 3 months with or without accompanying leg pain. ${ }^{26}$ Purposive sampling ${ }^{27}$ of eligible clients through Aboriginal Community Controlled Health Services (ACCHS) and Aboriginal community organisations was undertaken. Snowball sampling through 'word of mouth' among participants, their families and community networks augmented these strategies. ${ }^{27}$ Utilising community networks within a sampling strategy has been recommended in Aboriginal health research. ${ }^{28}$

Eligible participants were contacted either by IBL, an Aboriginal coinvestigator, or by an existing participant who explained the project. After the project had been described and following consent, arrangements were made for an extended discussion. Participants were advised they were free to withdraw from the study or withdraw their data with no consequences. Thirty-two Aboriginal men (21) and women (11) participated. Most participants were from Regiontown (16), then Goldstone (9) and then Desertedge (7). Participants ranged in age between 26 and 72 years; the majority were middle aged (see table 1). With the exception of 


\begin{tabular}{|c|c|c|c|c|}
\hline Location & Code & Gender & Age & Disability level \\
\hline \multirow[t]{16}{*}{ Regiontown } & $\mathrm{R} 1$ & M & 48 & Moderate \\
\hline & $\mathrm{R} 2$ & $M$ & 53 & Moderate \\
\hline & R3 & $\mathrm{F}$ & 48 & Mild \\
\hline & R4 & $M$ & 54 & High \\
\hline & R5 & M & 42 & High \\
\hline & $\mathrm{R} 6$ & $\mathrm{~F}$ & 53 & Mild \\
\hline & R7 & M & 49 & Moderate \\
\hline & R8 & $M$ & 68 & Mild \\
\hline & R9 & $M$ & 41 & Moderate \\
\hline & $\mathrm{R} 10$ & $\mathrm{~F}$ & 35 & Moderate \\
\hline & R11 & $\mathrm{F}$ & 53 & Moderate \\
\hline & $\mathrm{R} 12$ & $F$ & 31 & Mild \\
\hline & $\mathrm{R} 13$ & $M$ & 26 & Moderate \\
\hline & $\mathrm{R} 14$ & M & 68 & Mild \\
\hline & R15 & $F$ & 52 & Mild \\
\hline & R16 & M & 54 & High \\
\hline \multirow[t]{9}{*}{ Goldstone } & G1 & M & 62 & Mild \\
\hline & G2 & $\mathrm{F}$ & * & Mild \\
\hline & G3 & M & * & Mild \\
\hline & G4 & M & 48 & High \\
\hline & G5 & $M$ & 30 & Moderate \\
\hline & G6 & $M$ & 73 & Moderate \\
\hline & G7 & $F$ & 44 & Moderate \\
\hline & G8 & M & 58 & Mild \\
\hline & G9 & $M$ & 60 's & Mild \\
\hline \multirow[t]{7}{*}{ Desertedge } & D1 & $F$ & * & Moderate \\
\hline & D2 & M & 42 & High \\
\hline & D3 & $F$ & * & Moderate \\
\hline & D4 & $M$ & 44 & Mild \\
\hline & D5 & $F$ & 56 & Moderate \\
\hline & D6 & M & 35 & Mild \\
\hline & D7 & M & * & High \\
\hline \multicolumn{5}{|c|}{$\begin{array}{l}\text { *Not discussed during conversations. } \\
\text { —Mild level CLBP disability. } \\
\text { —Moderate level CLBP disability. } \\
\text { — High level CLBP disability. } \\
\text { CLBP, chronic low-back pain. }\end{array}$} \\
\hline
\end{tabular}

one participant, all had sought healthcare at some point for their CLBP.

\section{Procedures}

Consultation with 11 Aboriginal community leaders and four non-Aboriginal representatives from ACCHSs was undertaken prior to the study starting with feedback validating study cause and approach. Data collection was undertaken between 2007 and 2010. Ethical approval was granted by the Western Australian Aboriginal Health Ethics Committee and Curtin University Human Research Ethics Committee.

The primary data collection method was in-depth semistructured interviews using 'yarning', an Aboriginal cultural form of conversation involving the sharing and exchange of information between two or more people and requiring the researcher to 'develop and build a relationship that is accountable to Indigenous people participating in the research'. ${ }^{29}$ Interviews were conducted by IBL with a male or female Aboriginal coinvestigator and typically begun with a 'social yarn' in which investigators established or reaffirmed an interpersonal connection, before moving on to a 'research yarn'. ${ }^{29}$ Most interviews were informal; however, an interview schedule was developed to guide the research yarn. Interviews were conducted in English or Aboriginal English. The interview schedule included open-ended questions, (eg, 'tell us the story of your pain') used with prompts to explore underlying beliefs (eg, "what do you feel is causing your pain? How do you feel your pain will go in the future?'). Interviews lasted from $30 \mathrm{~min}$ to $2.5 \mathrm{~h}$ (typically longer than $1 \mathrm{~h}$ ). With the exception of three interviews, all were audio-recorded. Three interviews were not recorded due to recorder malfunction or because the investigators judged that commencing recording may have disrupted the flow of an important yarn. Hand-written notes were taken of nonrecorded interviews. Follow-up interviews were undertaken with 19 participants (described below). Interview data were augmented by field observations recorded in a research journal.

\section{Analysis}

Transcripts were imported into NVivo qualitative data analysis software. ${ }^{30}$ Transcribed interviews and interview notes were repeatedly read by all members of the research team so all were familiar with the data. Equal weight was given to verbatim and interview note transcripts in the analysis. An initial analysis was undertaken by IBL who had undertaken transcription. Initially, a process of 'describe-compare-relate' was undertaken. ${ }^{31}$ Related stories, statements, words and phrases related to participants' beliefs about CLBP were coded into common themes in NVivo and a back and forth process between data undertaken to substantiate or challenge identified themes. Initial summaries of the data were reviewed by members of the interprofessional research team (physiotherapy, Aboriginal health, public health medicine and anthropology), and Aboriginal coinvestigators to include perspectives, themes and issues that might not otherwise have been considered. This informed ongoing writing and reflection, a process integral to data interpretation and analysis. ${ }^{31} 32$

A deeper level of analysis was then undertaken by comparing data between people who were more and less disabled. Participants were classified as high, moderate or low levels of disability informed by the multidimensional classification of Dunn $e t a e^{3}$ and our analysis of the impacts of CLBP including effects on life participation and emotional well-being. ${ }^{34}$ Comparisons were also made between cases by examining participants of different disability levels, genders, towns, ages and across theme groupings by generating a series of matrices in NVivo. Patterns, similarities, contradictions and exceptions were identified and informed ongoing analysis. ${ }^{35}$ The synthesis of this analysis was again discussed among the research team.

Preliminary findings were discussed during follow-up interviews with 19 participants, including a description 
of the interpreted level of disability, to verify the accuracy of preliminary findings through member checking. ${ }^{35}$ Priority was given to those who were most disabled by CLBP. Otherwise there were no particular features of those who were able to be followed up (eg, location, sex and age). All participants able to be contacted corroborated the content of initial interviews and most of them elaborated upon initial data. Participants were not followed up if they moved away, did not attend the arranged follow-up appointment times or were unable to be contacted during the study period. Repeating the above steps by re-examining the findings against raw data and a second level of writing completed a second round of formal analysis. Refined written summaries circulated again to members of the research team for discussion, critique and a 'reality check' ${ }^{31}$ from which a finalised summary was developed.

\section{RESULTS}

This paper focuses on two primary beliefs that emerged: the believed cause of pain including the origins of these beliefs, and future beliefs ('looking to the future'). The relationship between these beliefs and disability was examined.

\section{Cause of pain}

More than half of the participants believed that there were one or more structural or anatomical problems of their spine that were responsible for the cause of their pain (table 2). The majority attributed pain to damage of the disc or wear and tear of the spine ('they gave me an x-ray of the lower back, and um... it's all worn down': G11). Although this belief was expressed by those of varying level of disability, this was most common to those who were highly disabled:

Well I got told by [medical specialist] that it might be a trapped nerve or, that was before I had my first MRI, and then they said no you've got lower lumbar ... and as I said it's just bone crunchin' on bone (R5: 42-year-old man with highly disabling CLBP)

With the exception of one individual the belief of an underlying structural-anatomical cause of pain originated following the advice from a healthcare practitioner such as a medical specialist, general practitioner (GP), physiotherapist or chiropractor:

And the physio and chiro were both saying that it could be a hint of arthritis so went and got xrays and I think it was a CAT scan or MRI I had done on my back and then they found out that it was arthritis in the L4, L5 vertebrae. And it hasn't been getting any better since. When I first found out they put me on prescription medicine. (R13: 26-year-old man with moderately disabling CLBP)

The results of spinal radiological imaging were central to what participants believed was the cause of their pain. For several men who were moderately or highly disabled, these explanations, based on radiological imaging findings, adversely affected their emotional well-being:

At first I felt a bit weird with them telling me I had arthritis and that. I thought it was a bit of a joke. Then they showed me the x-rays and that and MRI, cat scan, whatever it was, it was a bit depressing and a bit shocking being young and finding out you've got arthritis. It wasn't too good. (R13)

R5: on the lower part I've got no natural gel so it's bone crunchin' on bone... and I've got no coccyx bone too. $\mathrm{Hmm}$....that was after my last MRI

IBL: okay. How does that make you feel with your back?

R5: depressed. Depressing.

Some participants recounted a diagnosis they had been given many years previously, highlighting how some advice from a healthcare practitioner can have a long lasting influence on beliefs. For example, R6 discussed her belief of a 'slipped disc' after interactions with a medical specialist 30 years previously:

That doc said I had a slipped disc ... it all depends on how your movements or your lifting (R6: 55-year-old woman with mildly disabling CLBP)

The belief of an anatomical cause of pain was commonly associated with other negative beliefs about pain; such as the inevitable negative future consequences of

Table 2 Believed causes of pain (several participants expressed multiple causes)

\begin{tabular}{llll}
\hline Believed cause & High disability (number of participants) & Moderate disability & Mild disability \\
\hline Disc & 2 & 4 & 1 \\
Worn out/arthritis & 2 & 2 & 2 \\
Bones (eg, 'shrinkage', 'crunching') & 2 & & 1 \\
Crooked/out of shape & - & 2 & - \\
Muscles & - & 1 & 1 \\
Weight & - & 1 & 1 \\
Stress & - & 1 & - \\
Do not know & 1 & 3 & 3 \\
Did not identify ongoing cause & - & 1 & 3 \\
\hline
\end{tabular}


pain. These beliefs were pronounced, although not a unique feature, of those with higher levels of disability ('I've already got them [the damage]. It's there for the rest of my life': R11).

In a number of instances the belief of an anatomical/ structural vulnerability of the spine became ingrained when reinforced on multiple occasions. G4 recounted numerous interactions with healthcare practitioners that influenced his beliefs. In particular, information from a medical specialist and multiple spinal operations to address a structural/anatomical pathology powerfully reinforced a structural-anatomical explanation of his pain and a catastrophic future outlook:

Well the last, second operation I had, one of the doctors reckoned, you know that's it, there were about 3 doctors or 4 doctors, the bloke who'd done the operation, you other ones were.. he said 'listen, you know your back is just stuffed' he said. 'I wouldn't worry about going in there and digging around again'. (G4: 48-year-old man with highly disabling CLBP)

A reliance on radiological imaging for a diagnosis also had negative consequences for several men for whom these investigations failed to provide a satisfactory diagnosis. One man in a remote town attributed the lack of imaging findings to what he perceived as the poor quality of radiological equipment in the remote health clinic. A lack of imaging findings also resulted in frustration with the inference that the pain was not legitimate:

He [doctor] said 'nothin's here, he might be $\mathrm{f}^{* * * * *}$ going silly, he might have imagined all this pain down here', cause no-one's telling me anythin'. Is it up here [in the head]? Psychological or whatever you know? Is something wrong with me? (R7: 49-year-old man with moderately disabling CLBP)

A smaller number of participants discussed how they did not know the cause of their CLBP. The reasons varied and in some instances were dependent on the past interactions with healthcare practitioners. Several participants were unsure because of a lack of explanation and speculated as to possible causes ('Landed on my tail bone must have jarred it. I'm not sure: R12), while others disbelieved previous explanations they had been given by a healthcare practitioner due to the longevity of their pain:

I don't know if it's the muscles or bloody, actually the spine itself or.. wouldn't have the faintest idea.... the doctor has been saying it's just the tightness of your back. And I said it's gotta be something else cause I've been living with this pain since I was twenty five (D6: 35-year-old man with mildly disabling CLBP)

\section{Looking to the future}

The majority of people expressed a negative future outlook, believing that their pain would get worse (table 3). This belief was almost invariably influenced by interactions with a healthcare practitioner

Well my doctors tell me that as you get older it will start, cause I got all, like pins and screws and all that there now, and a few other things, yeah. And they reckon as you get older it will start getting worser. As you get older then you'll probably be in a wheelchair for life, or something like that. So the doctors told me (G5: 30-year-old man with moderately disabling CLBP)

\section{And R5 who was highly disabled}

And he (specialist) laid out the cards on the table, and said like you know, if you overdo it, you just, you know, you might eventually end up in a wheelchair for the rest of your life (R5: 42-year-old man)

As noted above, six people explicitly spoke of concern about 'ending up in a wheelchair', a sentiment attributed in each case to the advice from a doctor

It's the doc that said that, you know if I don't slow down or quit playing sports or whatever I could, you know, do more damage or end up in a wheelchair or whatever ... if I don't have sports I don't really have anything.. I feel bored, I don't have life. I'm itching to get back out on the field now. Yeah, it's just, what do I do? (R13: 26-year-old man with moderately disabling CLBP)

A common narrative shared by those who were more highly disabled was the presence of two coexisting beliefs; the perception of an anatomical/structural spinal problem, and a negative future outlook. In some cases this was also associated with a somewhat passive attitude towards management of pain and an apparent reliance on the medical system; for example, seeking a medical cure for the pain

well I'm hoping that they'll [medical specialists] make a decision, you know a compromise whether I'm going to get a fusion or cortisone (R5: 42-year-old man with highly disabling CLBP)

Four people held negative future beliefs without the belief there was an underlying structural or anatomical issue. In most cases, these individuals were unsure of the cause of their pain, based their opinion on a familial

\begin{tabular}{llll} 
Table 3 & Future beliefs about pain & \\
\hline & $\begin{array}{l}\text { Highly } \\
\text { disabled } \\
\text { (number of }\end{array}$ & $\begin{array}{l}\text { Moderately } \\
\text { disabled }\end{array}$ & $\begin{array}{l}\text { Mildly } \\
\text { disabled }\end{array}$ \\
Future & participants) & 8 & 3 \\
\hline Negative & 5 & 2 & 5 \\
Positive & - & 1 & 2 \\
Unsure & 1 & - & 2 \\
Stay same & - & 2 & 1 \\
Not discussed & - & &
\end{tabular}


history of back pain, or felt that a worsening of pain was inevitable with age

R7: well it'll get worse as you get older. I'm fifty next year.

IBL: yep, why's that?

R7: well because no-one can do anything about it and I can't do anything about it cause I don't know what's wrong with it

Seven people held a positive outlook about their future, believing that they would improve. In general these people were less disabled by pain (table 3). With the exception of one man, participants with a positive future outlook did not hold these views because of interactions with a healthcare practitioner. Rather, a positive future outlook was a self-held belief. Participants related their positive future belief to what they could do to selfmanage their pain, a viewpoint suggestive of higher self-efficacy:

I'm under the impression that if I lost weight I'll be right, you know. To me it's not major but I know it's there. I'm one of the ones that don't really let it get me down. (R3: 48-year-old woman with mildly disabling CLBP)

For one woman, a positive outlook had been reinforced by her previous successful experience of managing her pain

Years ago when I was quite active it was yeah, right (R10:

35-year-old woman with moderately disabling CLBP)

One man was an exception, having received advice from a healthcare practitioner that had contributed to a positive future outlook. His words highlight how advice from his GP, in addition to a positive experience of recovery, assisted a positive outlook

It's all right, doc reckons it's not that serious ... the longer it's going my back's getting better and better (D4:

44-year-old man with mildly disabling CLBP)

A small number of people were unsure how their pain would impact on their later lives. Two of the cause of their pain and recounted receiving ambiguous, and sometimes conflicting information, from healthcare practitioners or from family members. Two older men who were mildly disabled believed their pain would stay the same. These men had lived with CLBP in excess of 20 years suggesting the length of time they had lived with their symptoms influenced this belief.

\section{DISCUSSION}

This study found that Aboriginal men and women in a regional and two remote areas of Australia held predominantly negative beliefs about CLBP arising from healthcare interactions with medical specialists, general practitioners, physiotherapists and chiropractors. Negative beliefs were most common among those who more disabled and suggest that CLBP disability is partly iatrogenic. Strategies to improve CLBP beliefs must address the beliefs of those with CLBP as well as healthcare practitioners. Our findings demonstrate the far-reaching influences of biomedical approaches to CLBP including the powerful influence that healthcare practitioner beliefs and communication can have on a person with pain. These findings are a potential lesson for all populations exposed to Western biomedical approaches to examination and management of low back pain (LBP).

Our findings differ from previous research undertaken more than a decade ago that concluded that the cultural beliefs of Aboriginal people were such that CLBP was not viewed as a health issue and these protected against disabling CLBP ${ }^{14}$ In contrast to the previous research, one of the explicit aims of our study was to explore CLBP beliefs. Qualitative research methods and a focus on cultural security may have enabled a more accurate insight. Alternatively, the findings may represent an erosion of traditional beliefs due to greater exposure to biomedically orientated approaches for LBP. These findings highlight the potential of these approaches to increase disability via creating or reinforcing negative beliefs. We have previously reported how the maintenance of Aboriginal cultural beliefs and practices was integral to many participants' lives ${ }^{34}$; however, it appeared that CLBP beliefs were vulnerable to unhelpful Western influences. This raises concerns about the detrimental effects of biomedical oriented CLBP healthcare practices that dominate the management of LBP in Western societies. ${ }^{25}$ This may have important implications for the Westernisation of health systems around the world. For example addressing the emerging burden of CLBP among rural communities in developing countries ${ }^{36}$ must be considered carefully when there is the potential for Western biomedical approaches to increase disability.

Aboriginal participants most commonly expressed the belief that there was an anatomical/structural dysfunction causing their CLBP. Anatomical/structural beliefs of spinal dysfunction were invariably based on the findings of radiological imaging. Routine radiological imaging for LBP is not recommended by clinical guidelines because of the potential to cause more harm than good. ${ }^{37}{ }^{38}$ However overall, adherence to spinal imaging guidelines is known to be poor among healthcare practitioners highlighting that current practice is still dominated by negative biomedical beliefs, ${ }^{39}$ as was reported by participants in this study. A number of participants indicated that beliefs based on radiological imaging findings were longstanding. The longevity of these perceptions may be problematic as they may hold an inherent 'stickiness', are long lasting and may be resistant to change.

The confluence of different negative beliefs; that of an anatomical/structural vulnerability and the perception of a negative future outlook, distinguished those who were highly disabled. The perception of a negative future 
outlook has been reported to independently predict ongoing disability. ${ }^{6}{ }^{8}$ In our study it appeared that the combination of structural/anatomical beliefs, negative future perceptions, and the involvement of healthcare practitioners in the origins and reinforcement of these beliefs were critical. Conversely, participants who were less disabled held a more positive future outlook even when there was the perception of a structural/anatomical origin of pain. However with the notable exception of one individual, participants with a more positive future outlook did not hold this view because of advice they had received during healthcare; instead these were self-held beliefs. Our data supports the notion that individuals who are less disabled possess a 'resilient self-belief system' with a positive future outlook and employing more positive strategies to manage their pain. ${ }^{6}$ Again, these key findings reinforce the potential for biomedically based CLBP care to be highly detrimental. ${ }^{25}$

Concurrent strategies to address the beliefs of Aboriginal patients with CLBP and healthcare practitioners are needed. The need to address the gap between healthcare practice and evidence-based CLBP care is consistent with previous research. ${ }^{12}{ }^{13}$ For patients, addressing beliefs about CLBP are at the 'heart of the consultation'. ${ }^{40}$ Simple patient information targeting beliefs has been shown to improve beliefs and reduce disability among those with the most pronounced negative beliefs ${ }^{41}$ and providing tailored and culturally appropriate information that compliments clinical care is recommended by guidelines. ${ }^{42}$ The evidence from successful Aboriginal healthcare programmes has demonstrated that the process by which CLBP information is developed is important to the success of outcomes. The principles of collaboration and partnership between Aboriginal and non-Aboriginal staff, community involvement and control, building on identified community resources and non-Aboriginal staff who are culturally secure ${ }^{43} 44$ in part parallel the imperative for consumer participation in healthcare and for developing meaningful health information for consumers. ${ }^{45} 46$

A strength of this study was the extended engagement with Aboriginal participants resulting in a rigorous process of 'member check'. ${ }^{35}$ Although it was not possible to follow-up all participants the researchers are confident that all important issues were raised by those who did participate. Explicit attention to cultural security and engaging with people via their own beliefs and practices reinforced the cultural and ethical rigour of findings. Practical steps to ensure cultural security included consultation with Aboriginal people prior to starting the research, close collaboration with Aboriginal people within the research team, during data collection and analysis, and the use of culturally suitable research methods such as yarning. Other strengths were the multiple and contextually different sites in the study (regional and remote) and a cross discipline research team of non-Aboriginal and Aboriginal researchers from physiotherapy, Aboriginal health, public health medicine and anthropology. Although the sampling strategy was suitable and recommended in Aboriginal health research ${ }^{28}$ a limitation was that fewer women than men were interviewed meaning the issues for women may not have been fully represented. The imbalance possibly arose because Aboriginal women may have preferred a female researcher. ${ }^{47}$ IBL is male, although he worked closely with female coinvestigators. Cultural reasons such as "shame" ${ }^{48}$ may also have meant that women did not want to participate. The issue of social desirability is a potential limitation, with participants responding in a manner perceived favourable to the researchers. We believe this is unlikely due to the application of culturally secure research methods, attendance to language, a demedicalised approach using yarning and the frank disclosure of participants' during interviews.

\section{CONCLUSION}

This study provides evidence that negative beliefs about CLBP and its future consequences among Aboriginal people living in rural and remote areas of Western Australia are influenced by interactions with healthcare practitioners who communicate negative biomedical beliefs about LBP to their patients. These findings are consistent with research in other populations and support that disabling CLBP may be at least partly iatrogenic. The challenge for healthcare practitioners who work with people with LBP from any culture is to communicate in a way that builds positive beliefs about LBP and its future consequences, enhancing resilience to disability.

\section{Author affiliations}

${ }^{1}$ Combined Universities Centre for Rural Health, University of Western Australia, Geraldton, Western Australia, Australia

${ }^{2}$ School of Physiotherapy, Curtin University, Perth, Australia

${ }^{3}$ Combined Universities Centre for Rural Health, University of Western Australia, Geraldton, Western Australia, Australia and Geraldton Regional Aboriginal Medical Service, Geraldton, Western Australia, Australia ${ }^{4}$ School of Medicine, The University of Notre Dame Australia, Perth, Australia

${ }^{5}$ Anthropology and Sociology, The University of Western Australia, Perth, Australia

Acknowledgements We gratefully acknowledge the participation of Aboriginal people and communities in one regional and two remote Western Australian towns. We also acknowledge the considered input and assistance in data collection of Uncle Des Thompson, Gwen Rakabula, Eric Dalgety, Virginia Cameron and Patrick Cameron. We thank Fiona Stanley for her comments on an earlier draft of this paper.

Contributors IBL had the idea for the study and all authors collectively developed the study concept and design. IBL collected the data with listed collaborators. All authors contributed to data analysis. IBL drafted the initial manuscript and all authors contributed to ongoing writing and critical revision. All authors approved the final manuscript.

Funding This research received no specific grant from any funding agency in the public, commercial or not-for-profit sectors.

\section{Competing interests None.}

Ethics approval Western Australian Aboriginal Health Ethics Committee and Curtin University Human Research Ethics Committee.

Provenance and peer review Not commissioned; externally peer reviewed. Data sharing statement No additional data are available. 


\section{REFERENCES}

1. Gatchel RJ, Peng YB, Peters ML, et al. The biopsychosocial approach to chronic pain: scientific advances and future directions. Psychol Bull 2007;133:581-624.

2. Waddell $\mathrm{G}$. The back pain revolution. 2nd edn. Edinburgh: Churchill Livingstone, 2004.

3. Brault MW, Hootman J, Helmick CG, et al. Prevalence and most common causes of disability among adults-United States, 2005. Morb Mortal Wkly Rep 2009;58:421-6.

4. Katz JN. Lumbar disc disorders and low-back pain: socioeconomic factors and consequences. J Bone Joint Surg Am Vol 2006;88:21-4

5. Deyo RA, Mirza SK, Turner JA, et al. Overtreating chronic back pain: time to back off? J Am Board Fam Med 2009;22:62-8.

6. Foster NE, Thomas E, Bishop A, et al. Distinctiveness of psychological obstacles to recovery in low back pain patients in primary care. Pain 2010;148:398-406.

7. Vlaeyen JWS, Linton SJ. Fear-avoidance and its consequences in chronic musculoskeletal pain: a state of the art. Pain 2000;85:317-32.

8. Young Casey C, Greenberg MA, Nicassio PM, et al. Transition from acute to chronic pain and disability: a model including cognitive, affective, and trauma factors. Pain 2008;134:69-79.

9. Gross DP, Ferrari R, Russell A, et al. A population-based survey of back pain beliefs in Canada. Spine 2006;31:2142-5.

10. Ihlebaek C, Eriksen HR. Myths and perceptions of back pain in the Norwegian population, before and after the introduction of guidelines for acute back pain. Scand J Public Health 2005;33:401-6.

11. Moffett JAK, Newbronner E, Waddell G, et al. Public perceptions about low back pain and its management: a gap between expectations and reality? Health Expect 2000;3:161-8.

12. Bishop A, Foster NE, Thomas E, et al. How does the self-reported clinical management of patients with low back pain relate to the attitudes and beliefs of health care practitioners? A survey of UK general practitioners and physiotherapists. Pain 2008;135:187-95.

13. Buchbinder R, Staples M, Jolley D. Doctors with a special interest in back pain have poorer knowledge about how to treat back pain. Spine 2009;34:1218-26.

14. Honeyman PT, Jacobs EA. Effects of culture on back pain in Australian Aboriginals. Spine 1996;21:841-3.

15. Maher P. A review of 'traditional' Aboriginal health beliefs. Aust $J$ Rural Health 1999;7:229-36.

16. Lin IB, O'Sullivan PB, Coffin JA, et al. 'I am absolutely shattered': the impact of chronic low back pain on Australian Aboriginal people. Eur J Pain 2012;16:1331-41.

17. AlHW. Rural, regional and remote health: a guide to remoteness classifications. Canberra: Australian Institute of Health and Welfare, 2004.

18. Pink B, Allbon P. Health and welfare of Australia's Aboriginal and torres strait islander peoples. Canberra: Australian Institute of Health and Welfare and Australian Bureau of Statistics, 2008.

19. Crotty M. The foundations of social research: meaning and perspective in the research process. Crows: Nest Allen Unwin, 1998.

20. Jones S, Torres V, Arminio J. Negotiating the complexities of qualitative research in higher education: fundemental elements and issues. New York: Routledge, 2006.

21. Miller WT, Crabtree BF. Clinical research. In: Denzin NK, Lincoln YS, eds. Handbook of qualitative research. 2nd edn. London: Sage Publications, 2000;607-31.

22. Benner $P$. The tradition and skill of interpretive phenomenology in studying health, illness, and caring practices. In: Benner P, ed. Interpretive phenomenology. Thousand Oaks: Sage, 1994:99-127.

23. Coffin J. Rising to the challenge in Aboriginal health by creating cultural security. Aboriginal Islander Health Worker J 2007;31:22-4.

24. Guilfoyle A, Coffin J, Maginn P. 'Make sure there is a shady tree': participation and action research with Australian Aboriginal communities. In: Maginn P, Thompson SM, Tonts M, eds. Qualitative urban analysis: an international perspective. Oxford: Elsevier, 2008:213-40.
25. NHMRC. Values and ethics: quidelines for ethical conduct in Aboriginal and torre strait islander health research. Canberra: National Health and Medical Research Council, 2003.

26. WHO. The burden of musculoskeletal conditions at the start of the new millenium. Geneva: World Health Organization, 2003.

27. Sarantakos S. Sampling procedures. In: Sarantakos S, ed. Social research. Houndmills: Palgrave MacMillan, 2005:151-75.

28. Devitt J, McMasters A. Living on medicine: a cultural study of end-stage renal disease among Aboriginal people. Alice Springs: IAD Press, 1998.

29. Bessarab D, Ngandu B. Yarning about yarning as a legitimate method in indigenous research. Int $J$ Crit Indigenous Stud 2010;3:37-50.

30. NVivo 8 [program]. 8.0.335.0 SP4 version, 1999-2009.

31. Bazeley P. Analysing qualitative data: more than 'identifying themes'. Malays J Qual Res 2009;2:6-22.

32. Richardson L. Writing: a method of inquiry. In: Denzin NK, Lincoln YS, eds. Handbook of qualitative research. London: Sage Publications, 2000:923-48.

33. Dunn KM, Jordan K, Croft PR. Characterizing the course of low back pain: a latent class analysis. Am J Epidemiol 2006;163:754-61.

34. Lin I, O'Sullivan PB, Coffin J, et al. 'I am absolutely shattered': the impact of chronic low back pain on Australian Aboriginal people. Eur $J$ Pain 2012;16:1331-41.

35. Lincoln YS, Guba EG. Naturalistic inquiry. Beverley Hills: Sage, 1985

36. Muirden KD. Community oriented program for the control of rheumatic diseases: studies of rheumatic diseases in the developing world. Curr Opin Rheumatol 2005;17:153-6.

37. Rossignol M, Arsenault B, Dionne C, et al. Clinic on low-back pain in interdisciplinary practice (CLIP) guidelines. Montréal: Agence de la santé et des services sociaux de Montréal, 2007.

38. Webster SW, Cifuentes M. Relationship of early magnetic resonance imaging for work-related acute low back pain with disability and medical utilisation outcomes. J Occup Environ Med 2010;52:900-7.

39. French SD, Green S, Buchbinder R, et al. Interventions for improving the appropriate use of imaging in people with musculoskeletal conditions. Cochrane Database Syst Rev 2010(1) pp.CD006094.

40. Main CJ, Buchbinder R, Porcheret M, et al. Addressing patient beliefs and expectations in the consultation. Best Pract Res Clin Rheumatol 2010;24:219-25.

41. Burton AK, Waddell G, Tillotson KM, et al. Information and advice to patients with back pain can have a positive effect. A randomized controlled trial of a novel educational booklet in primary care. Spine 1999;24:2484-91.

42. NICE. Low back pain: early management of persistent non-specific low back pain. NICE clinical guideline 88. London: National Institute for Health and Clinical Excellence, 2009

43. NHMRC. Strengthening cardiac rehabilitation and secondary prevention for Aboriginal and Torres Strait Islander peoples: a guide for health professionals. Canberra: National Health and Medical Research Council, 2005.

44. Rowley KG, O'Dea K, Anderson I, et al. Lower than expected morbidity and mortality for an Australian Aboriginal population: 10 -year follow-up in a decentralised community. Med $J$ Aust 2008;188:283-7.

45. Hill S, Filippini G, Synnot A, et al. Presenting evidence-based health information for people with multiple sclerosis: the IN-DEEP project protocol. BMC Med Inform Decis Making 2012;12;20.

46. Hill S. The knowledgeable patient: communication and participation in heath. A Cochrane handbook. Chichester: Wiley- Blackwell \& The Cochrane Collaboration, 2011.

47. Toussaint S, Mak D, Straton J. Marnin business: anthropological interpretations of cervical screening among Australian Aboriginal women. Aust J Prim Health Interchange 1998;4:43-52.

48. Harkins J. Shame and shyness in the Aboriginal classroom. Aust $J$ linguist 1990;10:293-306. 